\title{
Off-the-Shelf Allogeneic CAR-T Cells or Other Immune Effector Cells
}

\author{
Stephane Depil and Waseem Qasim
}

"Off-the-shelf" allogeneic CAR TCR $\alpha \beta$ T cells and other immune effector cells, such as natural killer (NK) or gamma delta (gd) T cells, can be premanufactured from healthy donors and may offer alternatives to autologous strategies. However, major barriers, namely HLA disparity resulting in graft versus host disease (GvHD) and host-mediated rejection, must be addressed.

\section{Strategies to Avoid Graft Versus Host Disease (GvHD)}

\section{Genome Edited $\alpha \beta$ TCR-Deleted T Cells}

Strategies to reduce TCR $\alpha \beta$ activity have included the use of truncated dominant-

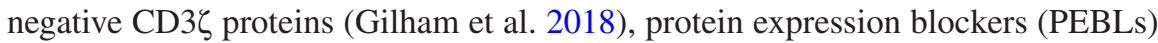
(Kamiya et al. 2018), small hairpin RNA (Bunse et al. 2014), and genome editing. Platforms for the latter have included zinc-finger nucleases (ZFN) homing endonucleases/meganucleases, transcription activator-like effector nucleases (TALEN), megaTALs, clustered regularly interspaced short palindromic repeat (CRISP/cas9), and base editors (BEs) (Depil et al. 2020). Clinical trials of universal TCR-depleted CAR19 T cells produced using TALENs (Servier/Allogene) have been published (Qasim et al. 2017; Benjamin et al. 2020), and the first applications of meganuclease

\footnotetext{
S. Depil $(\bowtie)$

Cancer Research Center of Lyon, Léon Bérard Cancer Center, University Claude Bernard Lyon 1, Lyon, France

e-mail: stephane.depil@lyon.unicancer.fr

W. Qasim

UCL Great Ormond Street Institute of Child Health, London, UK

e-mail:w.qasim@ucl.ac.uk 
(Precision Bio) and CRISPR engineering were recently reported (CRISPR Therapeutics). The manufacturing steps share common aspects of healthy donor $\mathrm{T}$ cell activation with anti-CD3/CD28 antibodies, editing by electroporation of nucleases and viral vector delivery of the CAR transgene. Depletion of residual TCR $\alpha \beta$ T cells using a magnetic bead column ensures that the carriage of potentially alloreactive T cells is kept below the threshold that might lead to GvHD.

\section{Virus-Specific T (VST) Cells}

Third-party, donor-derived VST cells have been investigated in allogeneic SCT and appear to induce reduced levels of GvHD, presumably due to their restricted, virusspecific, repertoire, and memory $\mathrm{T}$ cell phenotype. Examples include allogeneic EBV-specific T cells transduced to express CAR19 (Curran et al. 2011) and antiCD30 CAR (Savoldo 2007).

\section{Alternative Immune Effector Cells}

Immune effector cells not associated with induction of GvHD, including NK cells modified via lentiviral transduction to express CAR 19, exhibited early phase efficacy in CLL (Liu et al. 2020). Similarly, iNKT or $\gamma \delta T$ cells may have advantages against solid tumours, but clinical experience is still limited (Gentles et al. 2015). Engineering of CAR macrophages with antitumour properties has also been described recently (Klichinsky et al. 2020).

\section{Strategies to Avoid Host-Mediated Rejection of Allogeneic Immune Cells}

Beyond partial HLA matching of third-party donor cell banks, there are two main strategies to address the risk of host-mediated rejection.

\section{Resistance to Lymphodepletion and Immunosuppression}

CAR-T cells have been genome edited to become resistant to an anti-CD52 monoclonal antibody through disruption of CD52 (Benjamin et al. 2020). This approach has the advantage of suppressing all CD52+ immune cells that can mediate rejection, such as T, B, and NK cells, although prolonged immunosuppression is associated with a higher risk of serious virus reactivation. Engineering strategies have also been used to confer resistance to calcineurin inhibitors. Manufacturing steps can be multiplexed alongside editing of the TCR locus during electroporation or added to the vector design and incorporated into transduction steps. 


\section{Removal of HLA for Evading Host Immunity}

Removal of HLA class I molecules on the cell surface to avoid CD8 ${ }^{+} \mathrm{T}$ cell-mediated rejection can be achieved by disrupting the common beta 2-microglobulin gene. This approach is currently being investigated via multiplexed editing of CAR19 T cells (CRISPR Tx). In theory, the complete absence of HLA class I molecules may increase NK-mediated rejection through 'missing self' responses, and in modelling, this can be prevented by the expression of nonpolymorphic HLA molecules, such as HLA-E. Rejection through recognition of HLA class II on activated T cells may be addressed by disruption of critical transcription factors, such as CIITA and RFXANK (Depil et al. 2020).

\section{Manufacturing Aspects of 'Off-the-Shelf' CARs}

The majority of 'off-the-shelf' $\mathrm{T}$ cell therapies have utilized healthy donor peripheral blood mononuclear cells (MNCs) acquired via steady-state leukapheresis, although alternative sources, including whole blood and umbilical cord blood, may be suitable. In the future, immune effector cells may also be derived from induced pluripotent stem cells (iPSCs). Theoretically, a master iPSC cell line has an unlimited capability to self-renew and can be banked and used indefinitely. In general, manipulations must be performed in a clean room setting under GMP conditions with suitable licencing and regulatory approvals. Most cell gene transfer and genome engineering strategies require cells to be actively in mitosis to ensure open chromatin and accessible DNA, and activation steps are crucial early in the manufacturing process. Closed system culture and expansion is now routine, with automation reducing labour intensive aspects. High-quality viral vector preparations and improved electroporation steps for genome editing and supplies of stabilized mRNA have been critical. Cryopreservation in convenient dose formulations and efficient cold chain shipping and storage is an essential component of premanufactured 'off-the-shelf' therapies.

\section{Key Points}

- Off-the-shelf allogeneic CAR-T cells devoid of significant GvHD potential can be manufactured.

- $\quad \alpha \beta$ TCR-deleted CAR-T cells and CAR NK cells have successfully entered the early clinical trial phase.

- Several strategies have been developed to avoid host-mediated rejection of allogeneic effector cells. 


\section{References}

Benjamin R, et al. Genome-edited, donor-derived allogeneic anti-CD19 chimeric antigen receptor $\mathrm{T}$ cells in paediatric and adult B-cell acute lymphoblastic leukaemia: results of two phase 1 studies. Lancet. 2020;396(10266):1885-94.

Bunse M, et al. RNAi-mediated TCR knockdown prevents autoimmunity in mice caused by mixed TCR dimers following TCR gene transfer. Mol Ther. 2014;22(11):1983-91.

Curran KJ, et al. Validation of donor derived virus specific T-lymphocytes genetically modified to target the CD19 antigen for the treatment of relapsed leukemia. Mol Ther. 2011;19:S90.

Depil S, et al. 'Off-the-shelf' allogeneic CAR-T cells: development and challenges. Nat Rev Drug Discov. 2020;19(3):185-99.

Gentles AJ, et al. The prognostic landscape of genes and infiltrating immune cells across human cancers. Nat Med. 2015;21(8):938-45.

Gilham DE, et al. TCR inhibitory molecule as a promising allogeneic NKG2D CAR-T cell approach. J Clin Oncol. 2018;36(15_suppl):e15042-2.

Kamiya T, et al. A novel method to generate T-cell receptor-deficient chimeric antigen receptor $\mathrm{T}$ cells. Blood Adv. 2018;2(5):517-28.

Klichinsky M, et al. Human chimeric antigen receptor macrophages for cancer immunotherapy. Nat Biotechnol. 2020;38(8):947-53.

Liu E, et al. Use of CAR-transduced natural killer cells in CD19-positive lymphoid tumors. N Engl J Med. 2020;382(6):545-53.

Qasim W, et al. Molecular remission of infant B-ALL after infusion of universal TALEN geneedited CAR-T cells. Sci Transl Med. 2017;9(374):eaaj2013.

Savoldo B. Blood. 2007;110(7):2620-30. https://doi.org/10.1182/blood-2006-11-059139.

Open Access This chapter is licensed under the terms of the Creative Commons Attribution 4.0 International License (http://creativecommons.org/licenses/by/4.0/), which permits use, sharing, adaptation, distribution and reproduction in any medium or format, as long as you give appropriate credit to the original author(s) and the source, provide a link to the Creative Commons license and indicate if changes were made.

The images or other third party material in this chapter are included in the chapter's Creative Commons license, unless indicated otherwise in a credit line to the material. If material is not included in the chapter's Creative Commons license and your intended use is not permitted by statutory regulation or exceeds the permitted use, you will need to obtain permission directly from the copyright holder.

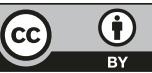

